Islam, S.I., et al., An In-silico analysis of the molecular interactions between PmCBP-VP24 and PmCBP-VP28 protein complex to understand the initial initiating events of shrimp WSSV infection International Journal of Life Sciences and Biotechnology, 2022. 5(2): p. 235-246. DOI: 10.38001/ijlsb.1055840

\title{
An In-silico analysis of the molecular interactions between PmCBP-VP24 and PmCBP-VP28 protein complex to understand the initial initiating events of shrimp WSSV infection
}

\author{
Sk Injamamul Islam ${ }^{*(D)}$, Moslema Jahan Mou $^{2}$, Saloa Sanjida $^{3}$, Sarower \\ Mahfuj $^{1}$ (i) Md. Ashraful Alam ${ }^{4}$ (D) Yeasmin Ara $^{\text {(iD }}$
}

\begin{abstract}
The White Spot Syndrome Virus (WSSV) has been the most pathogenic in the shrimp and other crustacean industries across the world in terms of production and financial benefits. Invertebrate vectors, freshwater, and sediments spread it horizontally as well as vertically from diseased broodstock to post-larvae. It is an exceedingly contagious disease that may cause entire mortality within 3-10 days of an outbreak under standard culture conditions. The sequencing and characterization of several WSSV strains have begun to reveal information regarding pathogen biology, pathogenicity, and WSSV must bind to the shrimp digestive system to infect it, and failure to do so results in ineffective infection. The Penaeus monodon chitin-binding protein (PmCBP), as well as the viral envelope proteins VP24 and VP28, are required for viruses to bind to the shrimp digestive system. In this study, we have shown the molecular interactions between PmCBP-VP24 and PmCBP-VP28 complex and speculated about the first steps of virus ingression in shrimps for the first time.
\end{abstract}

\author{
ARTICLE HISTORY \\ Received \\ 10 January 2022 \\ Accepted \\ 24 February 2022 \\ KEYWORDS \\ PmCBP, \\ VP24, \\ VP28, \\ molecular interaction, \\ WSSV
}

\section{Introduction}

In cultured penaeid shrimp, White Spot Syndrome Virus (WSSV) causes white spot disease (WSD). WSSV is more likely to infect penaeid shrimps like Litopenaeus vannamei, Penaeus

\footnotetext{
${ }^{1}$ Department of Fisheries and Marine Bioscience, Faculty of Biological Science, Jashore University of Science and Technology, Jashore-7408, Bangladesh

${ }^{2}$ Department of Genetic Engineering and Biotechnology, Faculty of Earth and Life Science, University of Rajshahi, Bangladesh

${ }^{3}$ Department of Environmental Science and Technology, Faculty of Applied Science and Technology, Jashore University of Science and Technology, Jashore-7408, Bangladesh

${ }^{4}$ Faculty, Rural Development Academy (RDA)-5842, Bangladesh

${ }^{5}$ Department of Fisheries Management, Hajee Mohammad Danesh Science and Technology University, Dinajpur

*Corresponding author: $\underline{678506331 @ \text { student.chula.ac.th }}$
} 
monodon, Marsupenaeus japonicus, and Fenneropenaeus indicus. WSSV is a big doublestranded DNA baculovirus, and infection with the pathogen in shrimp farming can result in up to $100 \%$ mortality in 3 to 10 days [1]. The white dots on the carapace of shrimp infected with WSSV are immediately identifiable [2]. The initial symptoms, on the other hand, arise after the animal has been infected and is near death. As a result, diagnosis methods have evolved from morphological-based characterization using electron microscopy (EM) to exceedingly effective immunological and molecular methods that previously could only detect the virus in asymptomatic carriers [3]. Given the severity of WSD in captive crustaceans, it is hardly unexpected that a lot of effort has gone into figuring out the disease's basic causes and finding potential remedies for disease control or mitigation[4]. Regardless of the diverse means adopted at present (i.e., environmental control, pre-exposure of shrimp to the pathogens, herbal treatments, DNA/RNA-based vaccines), there is no absolute drug or antiviral that can prevent the ingress of the virus. The main disadvantage of the illness is that its mechanism, which includes ingression, growth, and dispersion of the virus within the host, must be thoroughly understood [5]. The cannibalistic nature of shrimp results in infecting a healthy shrimp with WSSV. As a result, WSSV infection occurs when a normal shrimp feeds a WSSV-infected live/dead shrimp. The main site from which shrimp become infected is the lining of their digestive tube. Generally, chitinous linings line the esophagus, stomach, and hindgut while semipermeable peritrophic membranes (PM) line the midgut epithelium [5]. Chitin fibrils are embedded in a collage of proteins, proteoglycans, and mucopolysaccharides to form this PM. Several host cell membranes, particularly the cell membranes of epithelial cells, have receptors that can interact with virus envelope proteins and facilitate virus entry. An often-studied protein among all receptors in the chitin-binding protein $P m C B P(\mathrm{CBP}$ in Penaeus monodon). The interaction of yeast two hybrids with PmCBP has been studied in vitro using yeast two hybrids. VP24 and VP28 are two of the most significant proteins in this cluster of at least 11 viral envelope proteins associated with $\operatorname{PmCBP}[6,7]$. Furthermore, a unique complex protein aggregation (dubbed 'infectome') was discovered, which was made up of proteins such as VP24, VP28, VP31, VP32, VP39B, VP53A, and VP56 [7]. By being linked with $\mathrm{CBP}$, this complex performed a critical function in facilitating viral percolation over the basal membrane of the alimentary canal. Furthermore, the chitin-binding assays have 
revealed the association between infectomes and CBP is facilitated by VP24, VP28, and PmCBP [8]. The viral envelope protein VP24 protrudes outside from the viral envelope and this feature additionally facilitates its interaction with CBP [9, 10]. A critical factor for anchoring the viral particle to the inner lining of the shrimp alimentary canal is the interaction between chitin and VP24 within the stipulated period along with the association with VP28. Ineffective attachment advancement can lead to infection failure if this interaction is hampered. In this regard, the present study aims to use in-silico approaches to explore the molecular interactions between virus envelope proteins VP24, VP28, and shrimp receptor protein PmCBP. To accomplish this, 3D structures of $P m C B P$ were designed using molecular modeling. To understand the amino acid interactions occurring within VP24-PmCBP and VP28-PmCBP complexes, the docked 3D structure of PmCBP was subjected to molecular docking and dynamics approaches. The pmCBP-VP24 complex is vital while the PmCBPVP24 complex also aids in the entry of the virus into shrimp bodies. In one in-silico based study researchers predicted the interactions among the VP24-PmCBP complex [11], however, the interaction of $P m C B P$ with other virion proteins particularly with VP28 has not been studied yet. Moreover, the researchers only predict the binding interaction of VP24 proteins short amino acid sequence(186-200) with PmCBP [11]. Based on their study it is very difficult to conclude the initial instigation of WSSV. Because the three-dimensional structure of the VP24 has many active binding sites. Therefore, our study aimed to predict the interaction between PmCBP-VP24 and PmCBP-VP28 complex to provide information regarding the shrimp WSSV virus. Also, it is needed to understand the other virion protein's role with PmCBP protein to better analyze the WSSV initial action on the shrimp body. Thus, the present study provides a glimpse into the initial infection and the ingression of the virus within the shrimp body. Furthermore, this study may be used to search for inhibitors that can interfere with PmCBP's activity in forming complexes with VP24 and VP28 proteins, thereby inhibiting virus entry into shrimp. 


\section{Material and Methods}

\section{Sequence Analysis of PmCBP Protein}

The $P m C B P$ protein sequence was retrieved through a review of the literature [10]. The ProtParam program was used to calculate primary structure specifications for $P m C B P$ protein, including molecular weight, theoretical pI, atomic composition, extinction coefficient, estimated half-life, aliphatic index, and grand average of hydropathicity (GRAVY) [12]. The DiANNA tool was utilized to gain a more detailed understanding of the protein's secondary structure. The DiANNA tool is a neural network program that gives information about disulfide connectivity and protein fold stability. PmCBP protein disulfide bonds were accessed using the tool [13].

\section{Preparation of Molecules PmCBP, VP24, and VP28}

Homology modeling was used to predict the three-dimensional structure of $P m C B P$ employing BlastP and Protein Data Bank. Because an adequate template could not be found using BlastP, we used a de novo modeling strategy. Therefore, the three-dimensional structure of the target PmCBP protein was predicted using the Raptorx server (http://raptorx.uchicago.edu/) [14]. RaptorX is a protein structure prediction server developed by the $\mathrm{Xu}$ group, excelling at predicting 3D structures for protein sequences without close homologs in the Protein Data Bank (PDB). Given an input sequence, RaptorX predicts its secondary and tertiary structures as well as solvent accessibility and disordered regions. RaptorX also assigns the following confidence scores to indicate the quality of a predicted 3D model: P-value for the relative global quality, GDT (global distance test) and uGDT (un-normalized GDT) for the absolute global quality, and RMSD for the absolute local quality of each residue in the model. RaptorX-Binding is a web server that predicts the binding sites of a protein sequence, based upon the predicted 3D model by RaptorX. On the other hand, the 3D structure of VP24 was also predicted using the RaptorX server while the VP28 protein structure retrieves from Protein Data Bank (PDB) (2ED6). The models generated were visually analyzed using Pymol (Lill and Danielson, 2011). Although the VP24 has the 3D structure available in Protein Data Bank (PDB ID: 5HLJ), however the structure contains many other functional molecules. So, according to the objective of our 
study we decided to modelled the 3D structure of VP24 protein by RaptorX server to avoid the expanded ligand-protein interaction.

\section{Molecular Docking and Simulation Studies}

The molecular docking and simulation studies were carried out using Cluspro 2.0 web server. The algorithm running behind Cluspro 2.0 server is very robust and does not require any prior information regarding either template or binding site between the proteins. The online service can be accessed at (https://cluspro.bu.edu/login.php). The server provides a simple home page for basic use, requiring only two files in Protein Data Bank (PDB) format. However, ClusPro also offers several advanced options to modify the search; these include the removal of unstructured protein regions, application of attraction or repulsion, accounting for pairwise distance restraints, construction of homo-multimers, consideration of smallangle X-ray scattering (SAXS) data, and location of heparin-binding sites. Six different energy functions can be used, depending on the type of protein. Docking with each energy parameter set results in ten models defined by centers of highly populated clusters of lowenergy docked structures [15]. The final docked PmCBP-VP24 and PmCPBP-VP28 complex were visualized in Pymol and Discovery studio. Further, the amino acid interactions

occurring between protein PmCBP-VP24 and PMCBP-VP28 were tabulated using PDBsum [16]. The binding energy of the PmCBP-VP24 and PmCBP-VP28 complex was evaluated using the HawkDock server [17]. The server employs molecular mechanics PoissonBoltzmann surface area (MM-PBSA) to estimate interaction free energies between various protein-protein complexes [17].

\section{Results}

\section{Sequence Analysis of Protein PmCBP}

In the study, we performed sequence analysis of PmCBP using ProtParam, which revealed that $P m C B P$ was made of 168 amino acids. The estimated molecular weight of $P m C B P$ is 17471.11 Da. The total negatively charged residues and positively charged residues are 8 and 18 respectively. The Aliphatic index of the protein is 56.43 suggesting that it is a soluble protein. The Grand average of hydropathy (GRAVY) is - 0.114 suggesting that protein is 
hydrophilic. DiANNA tool predicted that the protein contained 3 disulfide linkages, which provided extracellular stability to the PmCBP (Table1).

Table 1 Physiochemical features of $P m C B P$ protein from different tools and server

\begin{tabular}{|l|l|}
\hline ProtParam tool & Values \\
\hline Number of AA & 168 \\
\hline MW & $17471.11 \mathrm{Da}$ \\
\hline pI & 4.45 \\
\hline Extinction coefficients & 7825 \\
\hline Instability index & 50.04 \\
\hline Aliphatic index & 56.43 \\
\hline GRAVY & -0.114 \\
\hline $\begin{array}{l}\text { Total number of negatively charged residues (Asp } \\
\text { + Glu) }\end{array}$ & 18 \\
\hline $\begin{array}{l}\text { Total number of positively charged residues (Arg } \\
+ \text { Lys) }\end{array}$ & 8 \\
\hline Formula & $\mathrm{C}_{775} \mathrm{H}_{1140} \mathrm{~N}_{198} \mathrm{O}_{253} \mathrm{~S}_{6}$ \\
\hline Total number of atoms & 2372 \\
\hline
\end{tabular}

Table 2 DIANA predict cysteine residues important in disulfide bonding

\begin{tabular}{|c|c|c|}
\hline \multicolumn{3}{|c|}{ DIANA } \\
\hline Predicted bonds & Distance & Bonded cysteine \\
\hline $86-126$ & 40 & STSFACLDRPY - QYSFLCGEGSR \\
\hline $101-139$ & 38 & DEENSCHIFHI - QKELTCVAESE \\
\hline $107-148$ & 41 & HIFHICYPALF - SEAIPCQESSN \\
\hline
\end{tabular}

\section{Preparation of Molecules PmCBP, VP24, and VP28}

The proportions of helix content, beta-sheet content, coil content, and overall confidence value were $0 \%, 1 \%, 18 \%, 82 \%$, and $72.5 \%$, respectively, according to the PROTEUS Structure Prediction Server 2.0 study. The final 3D structure of PmCBP was designed by the RaptorX server and out of 5 models we chose Model-1. Since model 1 depicted maximum RMSD score and $90.48 \%$ of the PmCBP residues show average 3D-1D score $\geq 0.2$ as per Verify3D results, therefore, it was finally selected as the best model for PmCBP (Figure 1). As predicted by the CASTp v.3.0 algorithm, the VP24 protein modeled contains 32 unique active sites (Figure 3). CASTp is a database server that can recognize regions on proteins, determine their boundaries, compute the area of the areas, and calculate the dimensions of 
the areas. Vacuums concealed within proteins and pockets on protein surfaces are also involved.

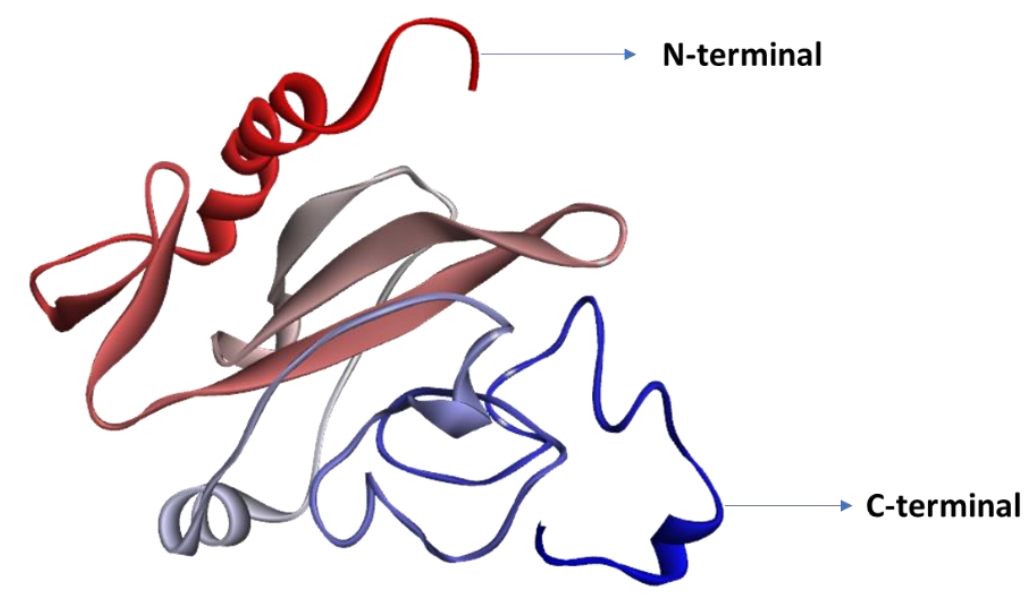

Fig 1 3D structure of PmCBP

Chain A

MHMWGVYA A I A GLTLILVVISIVVTNIELNKKLDKKDKDAYPVESEIINLTIN GVARGNHFNFVNGTLQTRNYGKVYVAGQGTSDSELVKKKGDIILTSLLGDGDHT LNVNKAESKELELYARVYNNTKRDITVDSVSLSPGLNATGREFSANKFVLYFKP TVLKKNRINTLVFGATFDEDIDDTNRHYLLSMRFSPGNDLFKVGEK

Fig 2 The active location of the VP24 protein. The active site of amino acid residues (Blue color)

\section{Molecular Docking and Simulation Studies}

The docking prediction performed by Cluspro 2.0 server result showed 10 models for each of the docked complexes. Model 1 (Figure $3(\mathrm{~A})$ ) is considered to be the most probable model of PmCBP-VP24 complex because it depicted RMSD (root-mean-square deviation) value $2.48 \AA$ whereas Model 7 (Figure 3 (B)) is considered to be the most probable model of PmCBP-VP28 complex which depicted RMSD value $2.53 \AA$. After the selection of these appropriate models, a detailed investigation was done into the amino acid sequences that interact between the protein PmCBP-VP24 and PmCBP-VP28 complex via the PDBsum web server. The amino acid interactions revealed that the PmCBP-VP24 complex is stabilized by 4 hydrogen bonds (Figure 3(A)). Within the PmCBP-VP24 complex, Ser81, Asn99, Gly48, 
and Ser81 of PmCBP respectively interact with Asn27, Thr26, Gly103, and Thr26 of VP24 through $\mathrm{H}$-bonds (Figure 3(A)). On the other hand, the amino acid interactions revealed that the PmCBP-VP28 complex is stabilized with $12 \mathrm{H}$-bonds. Within the PmCBP-VP28 complex, Asn51, Asn51, Asp49, Glu50, Glu50, Glu50, Asp75, Ile37, Thr41, Lys147, Ser152 and Ser153 of PmCBP respectively interact with His195, Thr197, Asn47, Thr46, Gly200, Thr201, Arg130, His40, His40, Glu109, Thr155 and Ser153 (Figure 3 (B)). The binding free energy of complex PmCBP-VP24 and $P m C B P-\mathrm{VP} 28$ was evaluated by the HawkDock server and found to be $-45.04(\mathrm{kcal} / \mathrm{mol})$ and $-48.07(\mathrm{Kcal} / \mathrm{mol})$ proving that the complex is stable. Additionally, some disulfide interactions also contribute towards the stability of the PmCBPVP24 complex (Figure. 3 (A, B)).

A

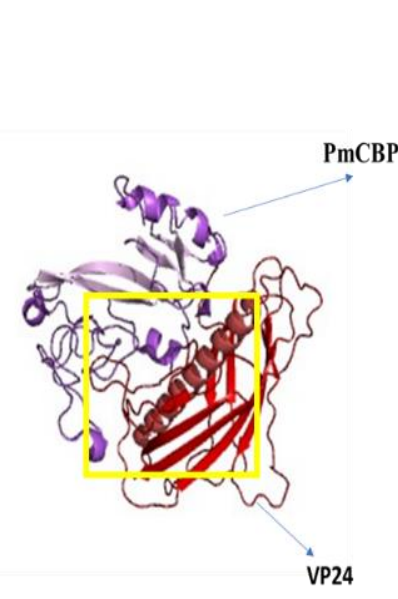

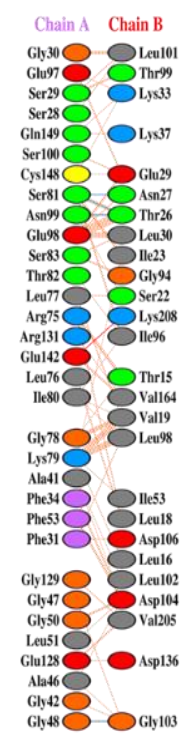

Key:

$\begin{array}{ll}\text { Salt } & \text { Disulphide } \\ \text { bridges } & \text { bonds }\end{array}$

- $\begin{aligned} & \text { Hydrogen } \\ & \text { bonds }\end{aligned} \quad$ แm. $\begin{aligned} & \text { Non-bonded } \\ & \text { contacts }\end{aligned}$

B
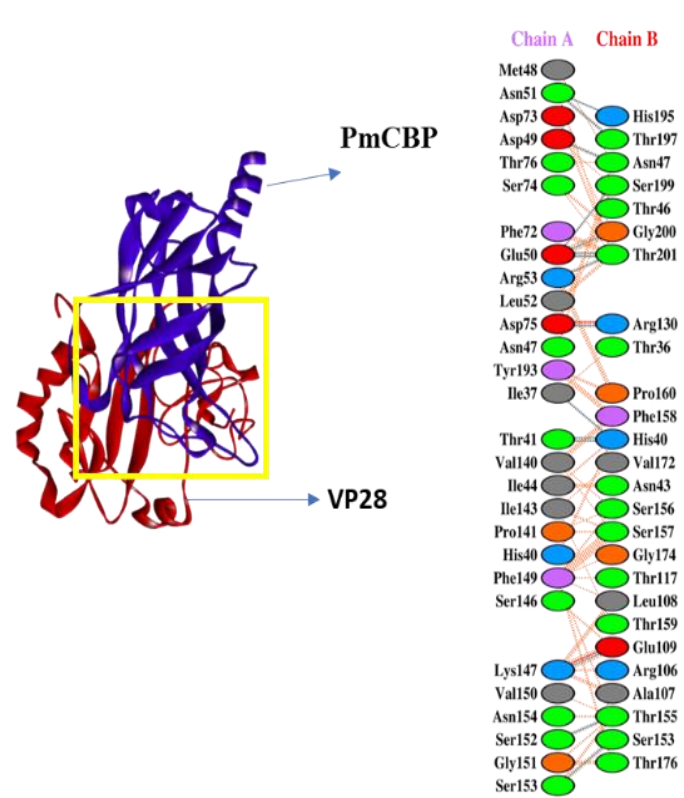

Fig 3 Protein-Protein interaction between (A) PmCBP-VP24 protein complex and (B) PmCBPVP28 protein complex 


\section{Discussion}

Protein-protein interactions are important to understanding the cellular function and organization of pathogens [15]. WSSV is primarily composed of its envelope and the proteins that are found within that envelop, which come in direct contact with shrimp digestive membranes and play a crucial role in the disease's propagation. WSSV envelope proteins were also considered capable of forming a protein complex dubbed an 'infectome'[18]. VP28 and VP24 are the most prevalent WSSV viral envelope proteins [19]. Previously, many studies revealed the role of Penaeus monodon Rab7 and chitin-binding protein-virion protein complex in WSSV infection through wet lab analysis [18, 20, 21]. Bioinformatics analysis showed stable interactions between VP24-PmCBP [11]. In their study, they only chose the amino acid sequence of VP24 from 186 to 200 to see the interaction with the PmCBP [11]. However, the interaction of the whole protein structure of VP24 with PmCBP protein needs to be brought into the light to better understand the initial instigation of WSSV infection. Moreover, the role and effect of VP28-PmCBP protein also can be the factor to analyze the initial infection of WSSV in shrimp.

To initiate infection with WSSV, dead or infected shrimp (viral particles) must be anchored properly to the shrimp alimentary canal's inner membrane. The virus traverses the shrimp alimentary canal to reach the basal membrane after accurate binding of its proteins with the shrimp receptor proteins that line the alimentary canal [5]. Upon reaching the basal membrane, the virion particles ooze into the alimentary canal before becoming plasma and eventually affecting the target organs such as eyestalks, cells of the cardiovascular system, brain, and reproductive organs such as gonads [22]. Individual shrimps become infected by the virus when it enters their nucleus and replicates itself to spread in the body of individual shrimps, resulting in their death [23]. Thus, the virus ingresses into the shrimp body when its receptor proteins make complexes with the viral envelope protein, which then anchors the virus to the shrimp. A vital role in disease initiation in shrimp is played by $P m C B P-\mathrm{VP} 24$ and $P m C B P-V P 28$, which are important protein complexes in the process. Despite having no structure for PmCBP, we designed it by in-silico approaches. In addition, VP24 and VP28 are believed to form the envelope protein complex, the so-called 'infectome', which plays a critical role in recognizing host cells, anchoring to them, and directing WSSV into the host 
cells [7]. Recent reports have shown that VP24 and VP28 interact with shrimp chitin receptors, but the exact role and molecular mechanisms involved remain unclear [24, 25]. Additionally, VP24 has been proposed as a potential monomertrimer transformation during the WSSV ingression mechanism in shrimp [9]. Therefore, when the virus enters the shrimp body, VP24 converts into its monomeric form and attaches itself to PmCBP's surface when its $\beta 9$ is exposed. Using this PmCBP-VP24 and PmCBP-VP28 complex, we discovered that the complexes are stabilized by hydrogen bonds and disulfide interactions for the first time. The H bonds located between Ser81, Asn99, Gly48, and Ser81 of PmCBP respectively with Asn27, Thr26, Gly103, and Thr26 of VP24, and these bonds might play a crucial role in adhering VP24 to PmCBP but they have to be further validated experimentally. Similarly, all the 12-hydrogen bonds between PmCBP-VP28 complexes also proved stable relations among them and this needs to be further researched as well. VP24 facilitates the attachment of VP28 to the host cell, thereby promoting membrane fusion to promote the viral infection after the formation of PmCBP-VP24 in the shrimp. VP28 attached to VP28 and PmCBP forms PmCBP-VP24, thereby promoting anchoring of WSSV to the shrimp's alimentary canal [9]. The infective amino acid residues found sandwiched between the complexes PmCBP-VP24 and PmCBP-VP28 in this work might be crucial for shrimp-viral interaction at the cellular level. In this way, disruption of the bonding between these amino acids could have a detrimental effect on PmCBP-VP24 and PmCBP-VP28 formation, thereby preventing the cascade of events that involve viral structural proteins, especially VP24 and VP28. This would lead to a slowed spreading of the virus throughout the body since the virus cannot transit seamlessly across the digestive tract. Given the importance of the complexes associated with WSSV ingression into shrimp, we will try to predict a potential inhibitor in our future studies that can disrupt the amino acid interactions in the complexes. If such inhibitors behave in a plausible antiviral manner in the wet lab, then they could be administered as a shrimp feed to disrupt the initial instigation process of the virus in shrimps, thus resulting in disease mitigation. 


\section{Conclusion}

WSSV genome and structure have been extensively studied, but information about how WSSV infects cells is limited. The purpose of this study was to establish the biological significance of the protein-protein interaction between WSSV envelope protein (VP24 and VP28) and Penaeus monodon chitin-binding protein (PmCBP). The WSSV needs to bind to shrimp digestive tracts that $P m C B P$ interacts with the viral envelope proteins, and failure to do so results in ineffective infection. Therefore, we found stable binding interactions between two major WSSV envelope proteins and $P m C B P$, and thus this study can play a vital role in producing WSSV drug inhibitors or vaccines in the future.

\section{Acknowledgments}

The author thanks Dr. Foysal Ahmed Sagore and Dr. Kazi Abdus Samad for helpful comments.

\section{References}

1. Oakey, J., et al., Global distribution of white spot syndrome virus genotypes determined using a novel genotyping assay. Archives of Virology, 2019. 164(8): p. 2061-2082.

2. Saravanan, K., et al., Investigation and confirmation of white spot syndrome virus (WSSV) infection in wild-caught penaeid shrimps of Andaman and Nicobar Islands, India. Virusdisease, 2017. 28(4): p. 368-372.

3. Curry, A., H. Appleton, and B. Dowsett, Application of transmission electron microscopy to the clinical study of viral and bacterial infections: present and future. Micron, 2006. 37(2): p. 91-106.

4. Vidya, R., P. Gireesh-Babu, and K. Pani Prasad, White spot syndrome virus Manipulates Ubiquitin Gene Expression in Penaeus monodon. Indian journal of virology : an official organ of Indian Virological Society, 2013. 24(1): p. 82-84.

5. Verma, A.K., et al., An update on mechanism of entry of white spot syndrome virus into shrimps. Fish Shellfish Immunol, 2017. 67: p. 141-146.

6. Verbruggen, B., et al., Molecular Mechanisms of White Spot Syndrome Virus Infection and Perspectives on Treatments. Viruses, 2016. 8(1): p. 23.

7. Huang, P.-Y., J.-H. Leu, and L.-L. Chen, A newly identified protein complex that mediates WSSV infection via chitin binding protein (CBP). The Journal of general virology, 2014. 95.

8. Verma, A.K., et al., An update on mechanism of entry of white spot syndrome virus into shrimps. Fish \& Shellfish Immunology, 2017. 67: p. 141-146.

9. Sun, L., et al., Crystal Structure of Major Envelope Protein VP24 from White Spot Syndrome Virus. Scientific Reports, 2016. 6: p. 32309.

10. Chen, K.Y., et al., Penaeus monodon chitin-binding protein (PmCBP) is involved in white spot syndrome virus (WSSV) infection. Fish Shellfish Immunol, 2009. 27(3): p. 460-5.

11. Yadav, K., et al., In Silico Prediction of Molecular Interaction Within PmCBP-VP24 Complex to Understand Initial Instigation of WSSV into Shrimps. Genetics of Aquatic Organisms, 2021. 5: p. 8793.

12. Wilkins, M.R., et al., Protein identification and analysis tools in the ExPASy server. Methods Mol Biol, 1999. 112: p. 531-52. 
13. Ferrè, F. and P. Clote, DiANNA: a web server for disulfide connectivity prediction. Nucleic Acids Res, 2005. 33(Web Server issue): p. W230-2.

14. Xu, J., M. McPartlon, and J. Li, Improved protein structure prediction by deep learning irrespective of co-evolution information. Nat Mach Intell, 2021. 3: p. 601-609.

15. Kozakov, D., et al., The ClusPro web server for protein-protein docking. Nature Protocols, 2017. 12(2): p. 255-278.

16. Laskowski, R.A., et al., PDBsum: Structural summaries of PDB entries. Protein Sci, 2018. 27(1): p. 129-134.

17. Weng, G., et al., HawkDock: a web server to predict and analyze the protein-protein complex based on computational docking and MM/GBSA. Nucleic Acids Res, 2019. 47(W1): p. W322-w330.

18. Huang, P.Y., J.H. Leu, and L.L. Chen, A newly identified protein complex that mediates white spot syndrome virus infection via chitin-binding protein. J Gen Virol, 2014. 95(Pt 8): p. 1799-1808.

19. Xie, X. and F. Yang, White spot syndrome virus VP24 interacts with VP28 and is involved in virus infection. J Gen Virol, 2006. 87(Pt 7): p. 1903-1908.

20. Sritunyalucksana, K., et al., PmRab7 is a VP28-binding protein involved in white spot syndrome virus infection in shrimp. J Virol, 2006. 80(21): p. 10734-42.

21. Chen, K.-Y., et al., Penaeus monodon chitin-binding protein (PmCBP) is involved in white spot syndrome virus (WSSV) infection. Fish \& Shellfish Immunology, 2009. 27(3): p. 460-465.

22. Lo, C.F., et al., Detection and tissue tropism of white spot syndrome baculovirus (WSBV) in captured brooders of Penaeus monodon with a special emphasis on reproductive organ. Diseases of Aquatic Organisms - DISEASE AQUAT ORG, 1997. 30: p. 53-72.

23. Escobedo-Bonilla, C., et al., A review on the morphology, molecular characterization, morphogenesis and pathogenesis of white spot syndrome virus. Journal of fish diseases, 2008. 31: p. 1-18.

24. Li, Z., et al., VP24 Is a Chitin-Binding Protein Involved in White Spot Syndrome Virus Infection. Journal of virology, 2015. 90.

25. Yi, G., et al., Vp28 of Shrimp White Spot Syndrome Virus Is Involved in the Attachment and Penetration into Shrimp Cells. Journal of biochemistry and molecular biology, 2004. 37: p. 726-34. 